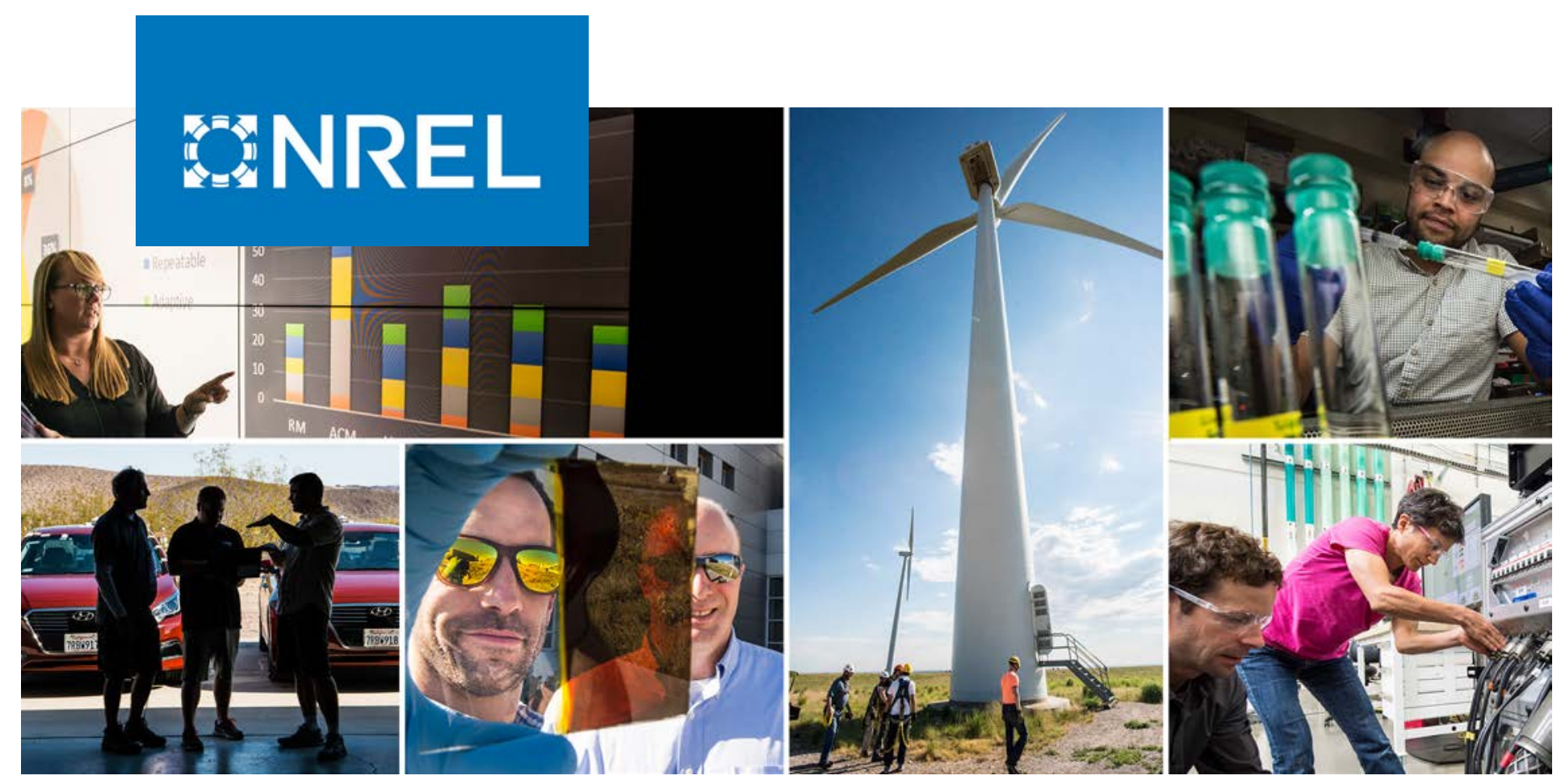

\title{
Adaptation of Commercial Current- Controlled Inverters for Operation with Virtual Oscillator Control
}

\section{Preprint}

Minghui Lu, ${ }^{1}$ Gab-Su Seo, ${ }^{2}$ Mohit Sinha, ${ }^{3}$

Fernando Rodriguez, ${ }^{4}$ Sairaj Dhople ${ }^{3}$ and Brian Johnson ${ }^{1}$

1 University of Washington

${ }^{2}$ National Renewable Energy Laboratory

${ }^{3}$ University of Minnesota-Twin Cities

${ }^{4}$ Enphase Energy

Presented at the 2019 IEEE Applied Power Electronics Conferences (IEEE PELS)

Anaheim, California

March 17-21, 2019

NREL is a national laboratory of the U.S. Department of Energy

Office of Energy Efficiency \& Renewable Energy

Operated by the Alliance for Sustainable Energy, LLC

This report is available at no cost from the National Renewable Energy Laboratory (NREL) at www.nrel.gov/publications.
Conference Paper

NREL/CP-5D00-72318

September 2019 


\title{
BNREL
}

\section{Adaptation of Commercial Current- Controlled Inverters for Operation with Virtual Oscillator Control}

\section{Preprint}

\author{
Minghui Lu, ${ }^{1}$ Gab-Su Seo, ${ }^{2}$ Mohit Sinha, ${ }^{3}$ \\ Fernando Rodriguez, ${ }^{4}$ Sairaj Dhople, ${ }^{3}$ and Brian Johnson ${ }^{1}$ \\ ${ }^{1}$ University of Washington \\ ${ }^{2}$ National Renewable Energy Laboratory \\ ${ }^{3}$ University of Minnesota-Twin Cities \\ ${ }^{4}$ Enphase Energy
}

\section{Suggested Citation}

Lu, Minghui, Gab-Su Seo, Mohit Sinha, Fernando Rodriques, Sairaj Dhople, and Brian Johnson. 2019. Adaptation of Commercial Current-Controlled Inverters for Operation with Virtual Oscillator Control: Preprint. Golden, CO: National Renewable Energy Laboratory. NREL/CP-5D00-72318. https://www.nrel.gov/docs/fy19osti/72318.pdf.

NREL is a national laboratory of the U.S. Department of Energy Office of Energy Efficiency \& Renewable Energy Operated by the Alliance for Sustainable Energy, LLC

This report is available at no cost from the National Renewable Energy Laboratory (NREL) at www.nrel.gov/publications.

Contract No. DE-AC36-08GO28308
Conference Paper

NREL/CP-5D00-72318

September 2019

National Renewable Energy Laboratory 15013 Denver West Parkway Golden, CO 80401

303-275-3000 • www.nrel.gov 


\section{NOTICE}

This work was authored in part by the National Renewable Energy Laboratory, operated by Alliance for Sustainable Energy, LLC, for the U.S. Department of Energy (DOE) under Contract No. DE-AC36-08GO28308. Funding provided by U.S. Department of Energy Office of Energy Efficiency and Renewable Energy Solar Energy Technologies Office. The views expressed herein do not necessarily represent the views of the DOE or the U.S. Government. The U.S. Government retains and the publisher, by accepting the article for publication, acknowledges that the U.S. Government retains a nonexclusive, paid-up, irrevocable, worldwide license to publish or reproduce the published form of this work, or allow others to do so, for U.S. Government purposes.

This report is available at no cost from the National Renewable Energy Laboratory (NREL) at www.nrel.gov/publications.

U.S. Department of Energy (DOE) reports produced after 1991 and a growing number of pre-1991 documents are available free via www.OSTI.gov.

Cover Photos by Dennis Schroeder: (clockwise, left to right) NREL 51934, NREL 45897, NREL 42160, NREL 45891, NREL 48097, NREL 46526.

NREL prints on paper that contains recycled content. 


\title{
Adaptation of Commercial Current-controlled Inverters for Operation with Virtual Oscillator Control
}

\author{
Minghui Lu*, Gab-Su Seo ${ }^{\dagger}$, Mohit Sinha ${ }^{\ddagger}$, Fernando Rodriguez ${ }^{\S}$, Sairaj Dhople ${ }^{\ddagger}$, and Brian Johnson* \\ ${ }^{*}$ Department of Electrical and Computer Engineering, University of Washington, Seattle, WA 98195, USA \\ ${ }^{\dagger}$ Power Systems Engineering Center, National Renewable Energy Laboratory, Golden, CO 80401, USA \\ $\ddagger$ Department of Electrical and Computer Engineering, University of Minnesota, Minneapolis, MN 55455, USA \\ $\S$ Enphase Energy, Austin, TX 78758, USA \\ Emails:mhlu@uw.edu,gabsu.seo@nrel.gov,sinha052@umn.edu, \\ frodriguez@enphaseenergy.com, sdhople@umn.edu,brianbj@uw.edu
}

\begin{abstract}
Virtual oscillator control (VOC) is a decentralized time-domain control technique for ac microgrids where inverters are regulated to emulate the dynamics of weakly nonlinear oscillators. VOC enables the design of modular and scalable systems where inverters can synchronize and share power without communication and in near real-time. In this paper, we show how off-the-shelf commercial inverters with current control can be reprogrammed to behave as voltage sources with virtual oscillator dynamics for deployment in islanded settings. We focus on commercial grid-tied inverters that have an inner current-control loop and show how the outer-loop controls can be straightforwardly modified to enable voltage-control-mode operation. To illustrate the practicality and ease of our approach, the proposed strategy was implemented on a $3.2 \mathrm{kVA}$ experimental test bed composed of 10 SunPower-brand micro-inverters with special firmware for VOC implementation. Results from the experiments not only demonstrate feasibility of the proposed dual-loop VOC architecture on a hardware setup but also show improved voltage regulation due to the additional voltage control loop.
\end{abstract}

\section{INTRODUCTION}

Multi-inverter microgrids (such as the one shown in Fig. 1) entail a multitude of technical challenges (e.g., stability, control design, voltage and frequency regulation) and have attracted considerable attention from both academia and industry [1]-[5]. To achieve communication-free synchronization among inverters, a majority of efforts have focused on droop control, which is a classical method that draws inspiration from synchronous machine systems [6]-[8], while other existing approaches [9], [10] control inverters to mimic the physical features of synchronous generators. On the other hand, departing from machine-based approaches in this application setting, virtual oscillator control (VOC) is a recently proposed control strategy, [4], [11]-[15], where inverters are programmed to emulate the dynamics of weakly nonlinear limit-cycle oscillators such as the Van der Pol (VDP) oscillator [16], [17]. It has been shown that VOC enables modular and scalable microgrids where synchronization, power sharing [4], as well as voltage and frequency regulation [18] are obtained without communication. Recent results also show that VOC subsumes the functionality of conventional droop control while provid- ing enhanced speed [11], [19], [20] due to its time-domain implementation.

At its core, the principle behind droop control is the enforcement of a linear relationship of measured real and reactive power with respect to frequency and voltage, respectively, at the inverter output terminals [11]. The distinctions between VOC and droop control have been characterized in [11], [19], where VOC provides enhanced synchronization dynamics over droop control due to its instantaneous power computation without the need for additional filters and averaging [11]. From an implementation standpoint, a dual loop structure consisting of outer voltage loop and inner current loop is commonly employed for droop controlled inverters to regulate output voltage characteristics [3], [21]. However, in [4], [11], [18]-[20], all prior implementations of VOC utilized conventional pulse width modulation (PWM) where the averaged switch terminal voltage was modulated to track the underlying oscillator dynamics directly. Although these initial implementations were simple, they came with the following disadvantages: i) diminished voltage regulation caused

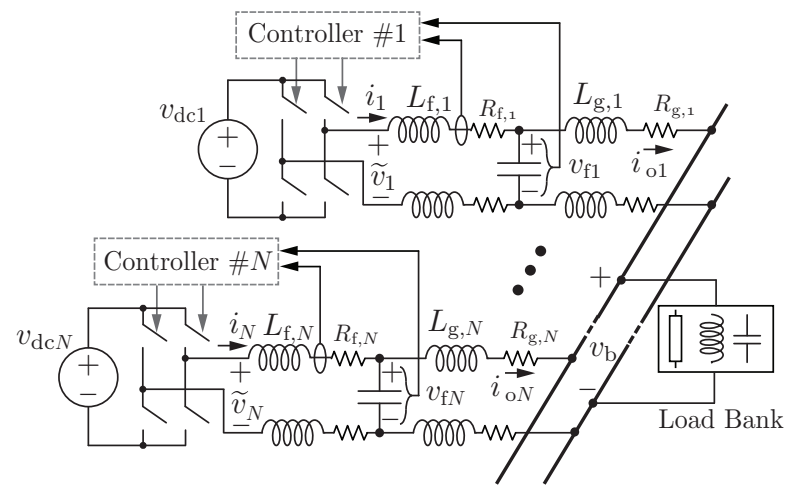

Fig. 1. Illustration of system architecture considered in this work: A modular system of $N$ parallel current-controlled inverters programmed to behave in grid-forming mode providing reliable power to local loads in the absence of a grid. 


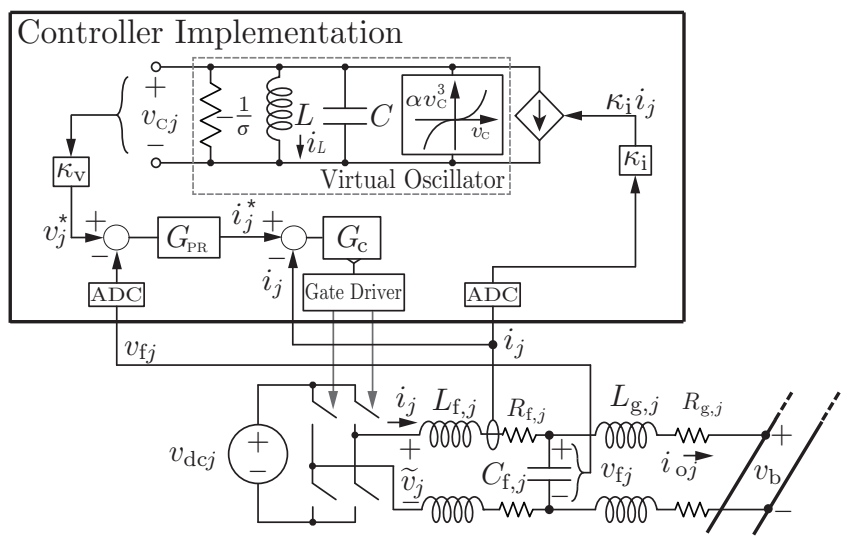

Fig. 2. The proposed dual-loop control architecture comprises a VDP oscillator, an outer voltage control loop, and an inner current controller. We illustrate details for the $j$ inverter above.

by output-filter voltage drops [18], ii) the virtual-oscillator controlled inverters can only be regulated as voltage sources, lacking compatibility with current controlled inverters, and iii) dependence on particular modulation schemes. In an attempt to bypass these limitations, a method was recently proposed in [22] that relied on a virtual inductance branch to generate a current command which was then fed to a downstream current controller. Although the method in [22] allowed for the use of VOC in current-controlled inverters, it does not allow for direct voltage control and departs from dual-loop structures which are known to have high bandwidth.

To address these issues while retaining compatibility with current controlled inverters, we propose an implementation where the VOC generates a voltage command which is then fed to a dual-loop control structure (which contains an outer voltage and inner current control loop). Specific control implementation and stability analysis are presented in Section II. The proposed dual-loop VOC implementation is experimentally validated in Section III. Concluding remarks and contributions are given in Section IV. Although the proposed control approach is generalized and applicable to many converter types, we restrict our focus to SunPower brand micro-inverters with an inner current control loop. The proposed method can be carried out with a straightforward firmware modification which allows the converter to retain key functionalities such as protection. Experimental results are obtained for a system of ten parallel connected micro-inverters with the proposed controller modification.

\section{A DuAl-Loop Structure to EnABle VOC IMPLEMENTATION}

In this section, we begin with an overview of the controller implementation, following which we discuss the system dynamical model. Finally, we outline an approach to establish synchronization of the dynamics.

\section{A. Controller Implementation}

Consider the multi-inverter system architecture in Fig. 1 which consists of $N$ parallel-connected inverters and a load.
Referring to Fig. 2, $L_{\mathrm{f}, j}$ and $R_{\mathrm{f}, j}$ are $j$-th inverter-side inductance and resistance, $L_{\mathrm{g}, j}$ and $R_{\mathrm{g}, j}$ are the grid-side inductance and resistance. Moreover, $i_{j}$ and $i_{\mathrm{o} j}$ are inverter-side and gridside currents, respectively. The proposed control architecture has the following subsystems: i) a VDP oscillator that generates an outer voltage loop command $v_{j}^{\star}$ and is implemented in digital form after discretization, ii) a capacitor voltage $v_{\mathrm{f} j}$ control loop that tracks the VOC voltage command with a proportional resonant $(\mathrm{PR})$ compensator, and iii) an inner current-control loop leading to a current-controlled inverter. Basically, in addition to the original VOC schemes in our previous publications [11], [18], the capacitor-voltage $v_{\mathrm{f} j}$ loop and inverter-side current $i_{j}$ loop have been implemented in the proposed architecture. Significantly, since each controller only requires locally available measurements, this yields a modular architecture with plug-and-play capabilities.

For the $j$-th inverter, the continuous time dynamics of the virtual-oscillator inductor current, $i_{L}$, and the voltage loop command, $v_{j}^{\star}$, are given by (see Fig. 2)

$$
\begin{aligned}
L \frac{d i_{L}}{d t} & =v_{\mathrm{c} j}=\frac{v_{j}^{\star}}{\kappa_{\mathrm{v}}}, \\
C \frac{d v_{j}^{\star}}{d t} & =-\alpha \frac{v_{j}^{\star 3}}{\kappa_{\mathrm{v}}^{2}}+\sigma v_{j}^{\star}-\kappa_{\mathrm{v}} i_{L}-\kappa_{\mathrm{v}} \kappa_{\mathrm{i}} i_{j} .
\end{aligned}
$$

Combining the two equations above, we get

$$
\frac{d^{2} v_{j}^{\star}}{d t^{2}}-\varepsilon \omega \sigma\left(1-\beta v_{j}^{\star 2}\right) \frac{d v_{j}^{\star}}{d t}+\omega^{2} v_{j}^{\star}=-\varepsilon \omega \kappa_{\mathrm{v}} \kappa_{\mathrm{i}} \frac{d i_{j}}{d t},
$$

where, we define constants $\varepsilon, \beta$, and resonant frequency, $\omega$, as follows:

$$
\varepsilon=\sqrt{\frac{L}{C}}, \quad \beta=\frac{3 \alpha}{\kappa_{\mathrm{v}}^{2} \sigma}, \quad \omega=\frac{1}{\sqrt{L C}} .
$$

As outlined in [4], the VOC parameters can be engineered such that the terminal voltages and system frequency stay within user-defined limits (see [18] for a comprehensive design procedure). A scaled value of the $j$-th virtual capacitor voltage serves as the reference for the downstream PR compensator, $v_{j}^{\star}=\kappa_{\mathrm{v}} v_{\mathrm{c} j}$. The PR compensator in turn regulates the output capacitor voltage, $v_{\mathrm{f} j}$. Lastly, the current controller regulates the converter-side inductor current, $i_{j}$, by modulating the power semiconductors.

Since we are interested in line frequency dynamics, the implicit modeling assumption is that the filter inductor current is equal to the output current, i.e., $i_{j} \approx i_{\mathrm{o}}$ when averaged over a switch cycle; and the reference gating signal is emulated at the terminals, i.e., $v_{\mathrm{f} j} \approx G_{\mathrm{C}}\left(i_{j}^{\star}-i_{j}\right)$.

\section{B. System Dynamic Model}

In the following, $x(s)$ denotes the Laplace-transform of the time-domain signal $x(t)$. Furthermore, define matrix $\Pi=$ $I_{N}-\frac{1}{N} 1_{N} 1_{N}^{\mathrm{T}}$, and note that entries of vector $\Pi x$ are the differences of individual entries of $x$ from the average of all entries of $x$. Finally, $I_{N}$ is the $N \times N$ identity matrix, and $1_{N}$ is a length- $N$ vector with all entries equal to unity. 


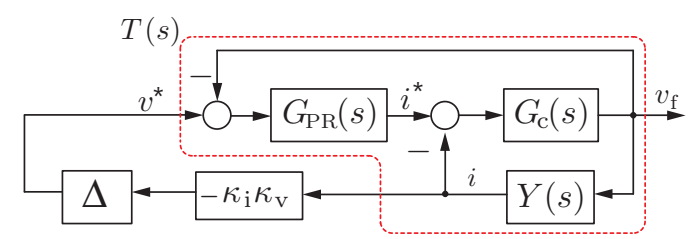

Fig. 3. Block diagram representation of the complete interconnected system.

Suppose $N$ inverters are connected in parallel to supply a resistive load, $R_{\text {load }}$. Let the vectors $y=v^{\star}=\left[v_{1}^{\star}, \ldots, v_{N}^{\star}\right]^{\mathrm{T}}$ and $i^{\star}=\left[i_{1}^{\star}, \ldots, i_{N}^{\star}\right]^{\mathrm{T}}$ collect the reference voltages and reference currents, respectively. A block diagram capturing controller and physical-side dynamics is shown in Fig. 3. In this block diagram, the PR controller is represented by transfer function $G_{\mathrm{PR}}(s)$ given by:

$$
G_{\mathrm{PR}}(s)=K_{\mathrm{P}}+\frac{K_{\mathrm{R}} s}{s^{2}+\omega^{2}},
$$

where $K_{\mathrm{P}}$ is the proportional gain, $K_{\mathrm{R}}$ is the resonant gain, and $\omega$ is the nominal frequency. Furthermore, $G_{\mathrm{C}}(s)$ represents the transfer function of the current controller, and $\Delta$ captures the nonlinear dynamics induced by the virtual oscillator circuits (2). In particular, denoting $x \in \mathbb{R}^{N}:=$ $\omega\left[\int_{0}^{t} v_{1}^{\star} d t, \ldots, \int_{0}^{t} v_{N}^{\star} d t\right]^{\mathrm{T}}, y \in \mathbb{R}^{N}:=v^{\star}=\left[v_{1}^{\star}, \ldots, v_{N}^{\star}\right]^{\mathrm{T}}$, system $\Delta$ encapsulates the following state-space model

$$
\begin{aligned}
& \dot{x}=\omega y, \\
& \dot{y}=-\omega x+H(y)-\varepsilon \omega \kappa_{\mathrm{v}} \kappa_{\mathrm{i}} i .
\end{aligned}
$$

Above, the entries of $H(y)$ are given by:

$$
[H(y)]_{j}=\varepsilon \omega \sigma\left(y_{j}-\frac{\beta}{3} y_{j}^{3}\right), j=1, \ldots, N .
$$

Finally, $Y(s)$ denotes the admittance matrix of the electrical network, and for a resistive load, $R_{\text {load }}$, it take the form:

$$
Y(s)=y_{g}(s)\left(I_{N}-\frac{y_{g}}{N y_{g}+R_{\mathrm{load}}^{-1}} 1_{N} 1_{N}^{\mathrm{T}}\right) \text {, }
$$

where $y_{g}(s)=\left(R_{g}+s L_{g}\right)^{-1}$.

\section{Stability Analysis}

To establish sufficient conditions for global asymptotic synchronization, we: i) first build an incremental system that looks at the dynamics of the terminal voltage differences, $\widetilde{v}_{j}$, among the inverters in time domain, ii) capture the electrical network description that relates the terminal voltages to the feedback current in frequency domain and then construct its state space realization to get a time-domain formulation, and iii) propose a quadratic Lyapunov function based on the states of the incremental system and the realized states of the electrical network system [4].

Multiplying $\Pi$ on both sides of (5), the state space description of the nonlinear oscillator subsystems in the incremental system is

$$
\begin{aligned}
& \Pi \dot{x}=\omega \Pi y, \\
& \Pi \dot{y}=-\omega \Pi x+\Pi H(y)-\varepsilon \omega \kappa_{\mathrm{v}} \kappa_{\mathrm{i}} \Pi i
\end{aligned}
$$

From the block diagram in Fig. 3, we derive the transfer function between $i(s)$ and $y(s)$. We begin by writing:

$$
\begin{gathered}
G_{C}(s) G_{\mathrm{PR}}(s)(Y(s) y(s)-i(s)) \\
=i(s)\left(I_{N}+Y(s) G_{C}(s)\right) .
\end{gathered}
$$

Isolating the map from $y(s)$ to $i(s)$ above, we get:

$$
\begin{aligned}
i(s) & =\left(I_{N}\left(1+G_{C} G_{\mathrm{PR}}\right)+Y(s) G_{C}\right)^{-1} G_{\mathrm{PR}} G_{C}(s) Y(s) y(s) \\
& =: T(s) y(s) .
\end{aligned}
$$

It can be verified that $\Pi T(s)=T(s) \Pi$. Therefore, $\Pi i(s)=$ $T(s) \Pi y(s)$. Thus, the incremental system can be equivalently seen as the feedback interconnection of the nonlinear oscillator dynamics, $\Delta$, and the linear system $T(s)$ (See Fig. 3). Next, we seek to leverage the properties of these subsystems to establish global asymptotic synchronization.

Let us assume $G_{C}(s)$ and $G_{\mathrm{PR}}(s)$ are designed taking $Y(s)$ into account such that $T(s)$ is positive real and therefore from the KYP lemma [16], for the minimal realization $(A, B, C, D)$, where $T(s)=C(s I-A)^{-1} B+D$, there exist matrices $P=$ $P^{\mathrm{T}} \succ 0, L$ and $W$ such that

$$
\begin{aligned}
P A+A^{\mathrm{T}} P & =-L^{\mathrm{T}} L, \\
P B & =C^{\mathrm{T}}-L^{\mathrm{T}} W, \\
W^{\mathrm{T}} W & =D+D^{\mathrm{T}} .
\end{aligned}
$$

The corresponding state-space representation is given by:

$$
\begin{aligned}
\dot{\xi} & =A \xi+B \Pi y, \\
\Pi i & =C \xi+D \Pi y .
\end{aligned}
$$

Now for the combined system (8)-(12), consider the potential function

$$
V=(\Pi x)^{\mathrm{T}} \Pi x+(\Pi y)^{\mathrm{T}} \Pi y+\frac{1}{2} \varepsilon \kappa_{\mathrm{v}} \kappa_{\mathrm{i}} \xi^{T} P \xi .
$$

The time rate of change of the potential function is given by:

$$
\begin{aligned}
\dot{V} & =(\Pi y)^{\mathrm{T}} \Pi H(y)-\varepsilon \kappa_{\mathrm{v}} \kappa_{\mathrm{i}}(\Pi y)^{\mathrm{T}} \Pi i \\
& +\frac{1}{2} \varepsilon \kappa_{v} \kappa_{i}\left(\xi^{\mathrm{T}}\left(P A+A^{\mathrm{T}} P\right) \xi+2 \xi^{\mathrm{T}} P B \Pi y\right) \\
& \leq-\frac{\varepsilon \kappa_{\mathrm{v}} \kappa_{\mathrm{i}}}{2}\left[\begin{array}{c}
\xi \\
\Pi y
\end{array}\right]^{\mathrm{T}} \underbrace{\left[\begin{array}{cc}
L^{\mathrm{T}} L & L^{\mathrm{T}} W \\
W^{\mathrm{T}} L & W^{T} W-\frac{2 \sigma \omega}{\kappa_{\mathrm{v}} \kappa_{\mathrm{i}}} I_{N}
\end{array}\right]}_{=: E}\left[\begin{array}{c}
\xi \\
\Pi y
\end{array}\right] .
\end{aligned}
$$

If the gains $\kappa_{\mathrm{v}}$ and $\kappa_{\mathrm{i}}$ are chosen such that matrix $E$ is positive semidefinite, we can invoke Lyapunov and LaSalle arguments to conclude global asymptotic synchronization.

\section{HARDWARE VALIDATION}

In this section, we describe the microgrid test-bed setup and present experimental results to validate the concept. 


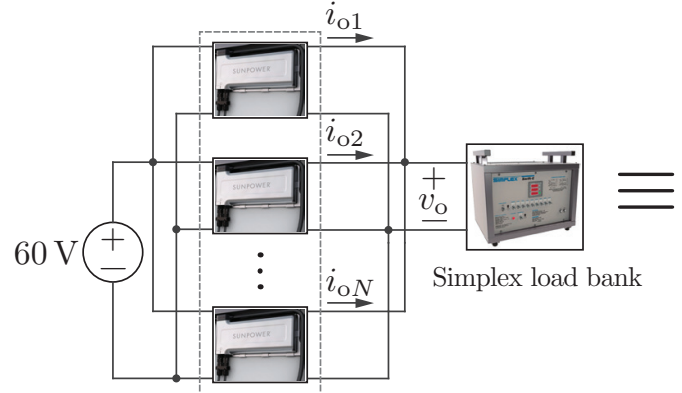

10 SunPower microinverters

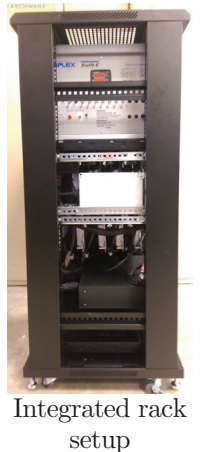

Fig. 4. Experimental setup consisting of 10 SunPower brand micro-inverters and a load bank. The micro-inverters were programmed with the proposed dual-loop control scheme illustrated in Fig. 2.

\section{A. Microgrid Testbed Setup}

To demonstrate feasibility of the proposed approach, a $3.2 \mathrm{kVA}$ microgrid testbed is constructed. As shown in Fig. 4, 10 SunPower brand micro-inverters, powered by a $60 \mathrm{~V} / 83 \mathrm{~A}$ AMETEK dc power supply, are connected in parallel to supply a variable resistive load. In particular, we realize the resistive load with a $5 \mathrm{~kW}$ Simplex brand load bank. The micro-inverters communicate with a PC through a power line communication (PLC) module in the system. The PLC module broadcasts inverter command signals, i.e., turn-on/off commands. Using these control commands emulating control signals from a higher-level controller, e.g., a system operator, the test-bed can demonstrate microgrid operation under different conditions. (Note that the communication between the micro-inverters and PC is only used to broadcast highlevel on/off signals; no high fidelity communication is needed for actual inverter operation.) The commercial inverter control firmware is modified to implement the VOC dynamics with the outer voltage PR compensator and inner current controller. Relevant control parameters are shown in Table I. The VOC parameters are designed based on the design procedure in [18] to implement $5 \%$ droop characteristics $(V: P$ and $\omega: Q$ droop is used in this testbed).

\section{B. Experimental Results}

Using the microgrid testbed, the key functionalities of VOC are validated on commercial hardware. In particular, we focus

TABLE I

CONTROL PARAMETERS

\begin{tabular}{c|l}
\hline Variables & Values \\
\hline \hline Scaling Factors & $\kappa_{\mathrm{V}}=240 \mathrm{~V} / \mathrm{V}, \kappa_{\mathrm{i}}=0.15 \mathrm{~A} / \mathrm{A}$ \\
\hline System Frequency & $\omega=2 \pi 60 \mathrm{rad} / \mathrm{s}$ \\
\hline PR controller & $K_{\mathrm{P}}=0.1 \mathrm{~A} / \mathrm{V}, K_{\mathrm{R}}=0.005 \mathrm{~A} \cdot \mathrm{s} /(\mathrm{V} \cdot \mathrm{rad})$ \\
\hline Oscillator Parameters & $\varepsilon=0.015 \Omega, C=0.1763 \mathrm{~F}, L=39.90 \mu \mathrm{H}$ \\
\hline Oscillator Parameters & $\alpha=7.198 \mathrm{~A} / \mathrm{V}^{3}, \sigma=10.80 \Omega^{-1}$ \\
\hline
\end{tabular}

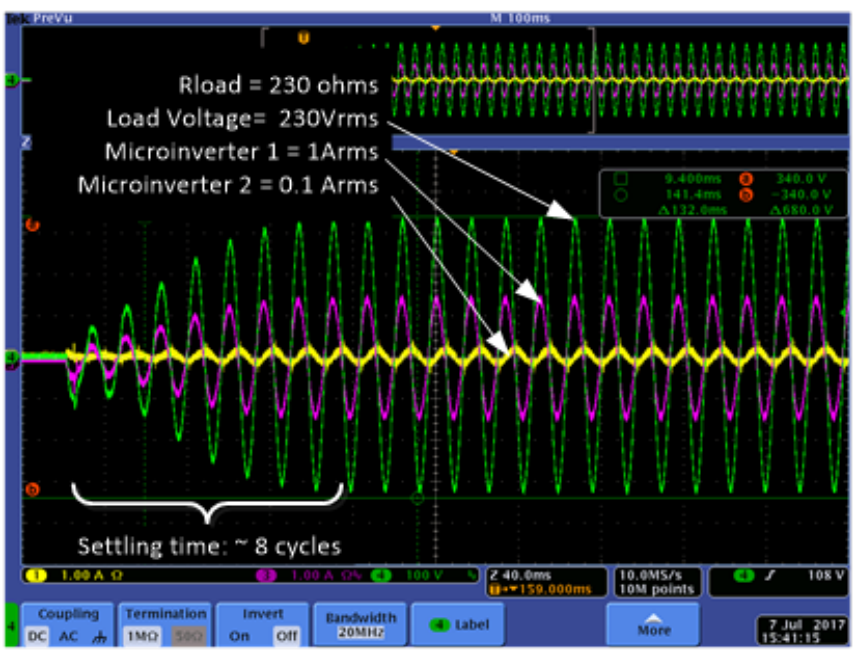

Fig. 5. Black start operation with a VOC inverter. Ch1: $i_{\mathrm{og} 2}$, Ch3: $i_{\mathrm{og} 1}$, Ch4: $v_{\mathrm{og}}$.

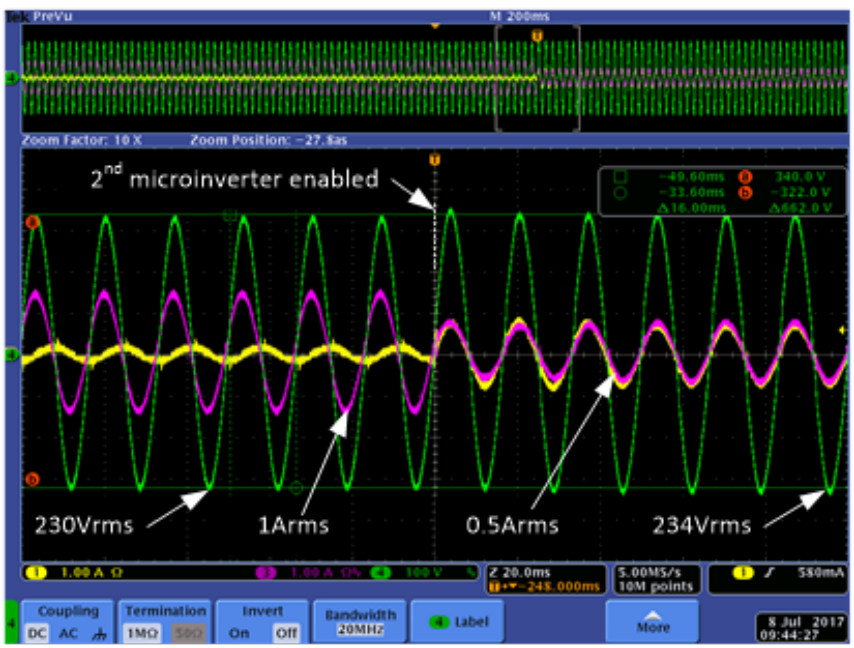

Fig. 6. Dynamic synchronization and load sharing of VOC inverters. Ch1: $i_{\mathrm{o} g 2}, \mathrm{Ch} 3: i_{\mathrm{o} g}, \mathrm{Ch} 4: v_{\mathrm{o} g}$

on system black start, addition and removal of units, and transients induced by load steps.

1) Black start Functionality: Black start functionality of grid forming inverters is critical for system resiliency in inverter-based systems [23]. Fig. 5 shows black start operation of the VOC inverters under a $250 \mathrm{~W}$ load. In this experiment, two VOC inverters are connected to the system while only inverter\# 1 is commanded to black start. Programmed with oscillator-based controls, the VOC inverter gradually increases its output voltage and regulates the grid voltage based on its dynamic and embedded droop characteristics (see details in [18]). As the output of inverter\#2 (which is inactive in this condition is also connected to the system in parallel), reactive power demand resulting from its $L C L$ filter is satisfied by the inverter\# 1 as shown in Fig. 5. 


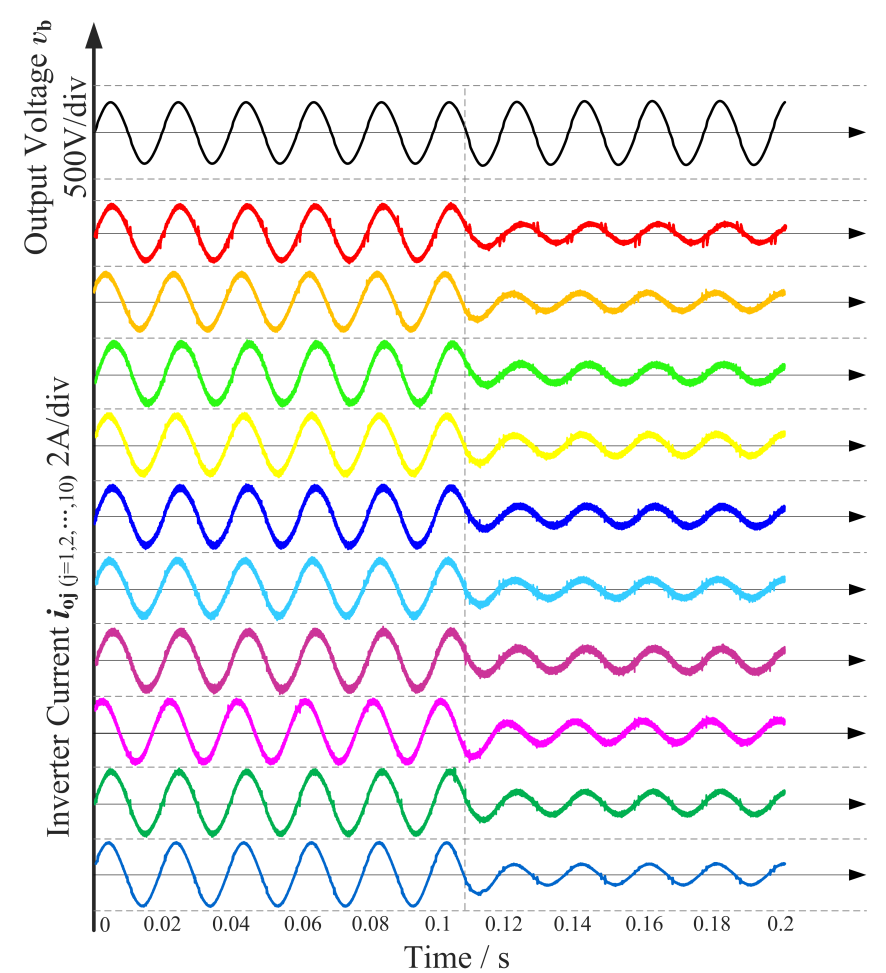

Fig. 7. Experimental measurements of bus voltage and current waveforms during load step-down $(3 \mathrm{~kW}$ to $1 \mathrm{~kW})$.

2) Unit addition and removal: To obtain a modular and decentralized system, it is necessary to show that grid-forming VOC inverters can be added and removed from the system. Utilization of a pre-synchronization scheme [18], the VOC inverters can be dynamically added to an energized system. Fig. 6 demonstrates dynamic operation when an additional inverter is added to the system. After it is activated by a PLC command, inverter \# 2 smoothly synchronizes to the microgrid. After the transients subside and the inverters are synchronized, the two inverters collectively operate to regulate the microgrid voltage and share the load. Note that, as the effective load is reduced to half, voltage is increased to $234 \mathrm{~V}$ by the droop law embedded in VOC. Due to the presence of the dual inner-loop controllers, the system retains the voltage defined by the outerloop VOC without $L C L$ filter voltage drops. Since excessive voltage drops may lead to errors in load sharing, it is clear that the dual-loop structure not only enhances voltage regulation but also improves power sharing.

3) Load steps: Figures 7-8 demonstrate system operation with ten grid-forming inverters under load transients. Figure 7 captures a step-down transient from $3 \mathrm{~kW}$ to $1 \mathrm{~kW}$. As the load decreases the ten VOC inverters adjust their output current to regulate the grid voltage. Fig. 8 demonstrates a load stepup from $1 \mathrm{~kW}$ to $3 \mathrm{~kW}$. In summary, measurements show that the system maintains a rapid response, current sharing, and a regulated bus voltage during load step transients between $1 \mathrm{~kW}$ and $3 \mathrm{~kW}$.

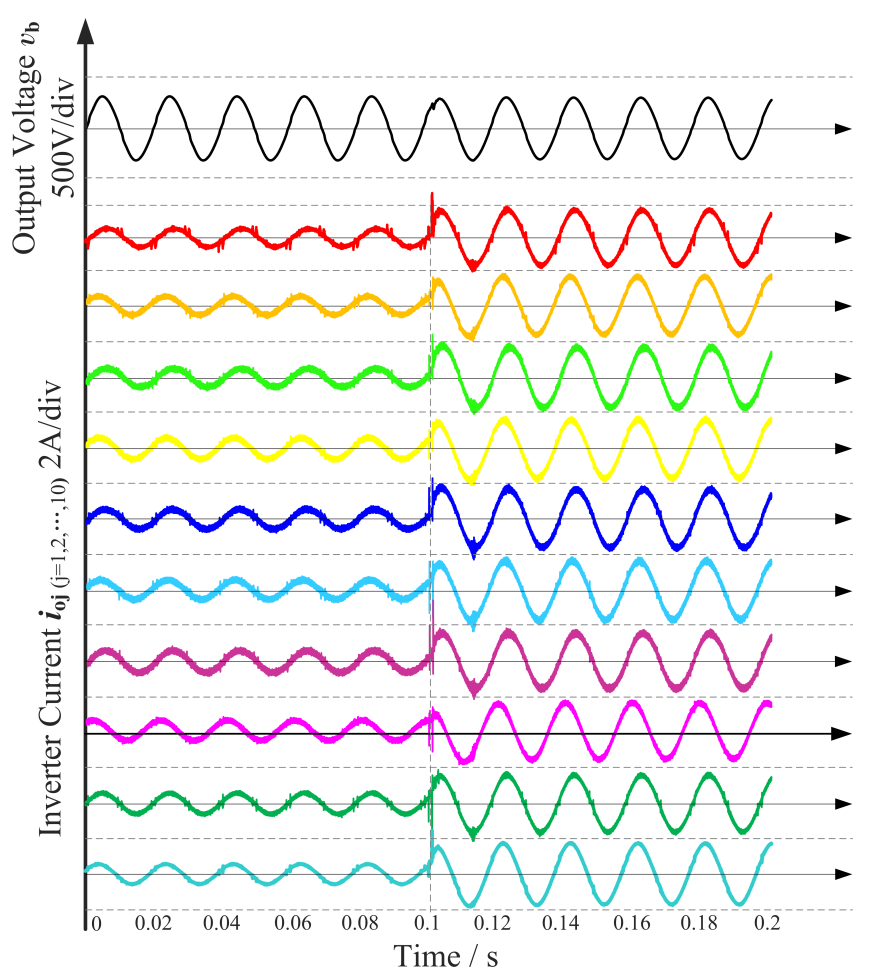

Fig. 8. Experimental measurements of bus voltage and current waveforms during load step-up ( $1 \mathrm{~kW}$ to $3 \mathrm{~kW})$.

\section{CONCLUSIONS}

In this paper, we propose a dual-loop control architecture that allows inverters with current controllers to be re-purposed for voltage control mode operation with VOC. In the proposed setup, the VOC voltage acts as a command for an outer voltage-control loop, which in turn actuates an inner currentcontrol loop. Not only does this extend the applicability of the VOC to settings where a current control is used, but it also improves voltage regulation by compensating for any output filter voltage drops. The dual loop structure was implemented on off-the-shelf SunPower brand photovoltaic micro-inverters. Experimental waveforms from a system of ten micro-inverters demonstrate communication-free current sharing, high bandwidth performance, and applicability to commercial hardware. Future work will be focused on numerical methods that incorporate the analysis in Section II and the condition in (15) in particular.

\section{ACKNOWLEDGEMENTS}

This work was supported in part by the National Renewable Energy Laboratory, operated by Alliance for Sustainable Energy, LLC, for the U.S. Department of Energy (DOE) under Contract No. DE-AC36 - 08GO28308. Funding was also provided by the DOE Office of Energy Efficiency and Renewable Energy Solar Energy Technologies Office under grant No. DE-EE0000 - 1583. The views expressed in the article do not necessarily represent the views of the DOE or the 
U.S. Government. Funding was also provided by the National Science Foundation through grants 1453921 and 1509277.

\section{REFERENCES}

[1] M. Chandorkar, D. Divan, and R. Adapa, "Control of parallel connected inverters in standalone AC supply systems," IEEE Transactions on Industry Applications, vol. 29, pp. 136-143, Jan./Feb. 1993.

[2] N. Pogaku, M. Prodanovic, and T. C. Green, "Modeling, Analysis and Testing of Autonomous Operation of an Inverter-Based Microgrid," IEEE Transactions on Power Electronics, vol. 22, pp. 613-625, Mar. 2007.

[3] A. Bidram and A. Davoudi, "Hierarchical Structure of Microgrids Control System," IEEE Transactions on Smart Grid, vol. 3, pp. 1963 1976, Dec. 2012.

[4] B. B. Johnson, S. V. Dhople, A. O. Hamadeh, and P. T. Krein, "Synchronization of Nonlinear Oscillators in an LTI Electrical Power Network," IEEE Transactions on Circuits and Systems I: Regular Papers, vol. 61, pp. 834-844, Mar. 2014.

[5] C. Arghir, T. Jouini, and F. Dörfler, "Grid-forming control for power converters based on matching of synchronous machines," Automatica, vol. 95, pp. 273-282, Sep. 2018.

[6] J. M. Guerrero, J. Vasquez, J. Matas, M. Castilla, and L. de Vicuna, "Control Strategy for Flexible Microgrid Based on Parallel LineInteractive UPS Systems," IEEE Transactions on Industrial Electronics, vol. 56, pp. 726-736, Mar. 2009.

[7] J. M. Guerrero, J. C. Vasquez, J. Matas, L. G. de Vicuna, and M. Castilla, "Hierarchical Control of Droop-Controlled AC and DC MicrogridsA General Approach Toward Standardization," IEEE Transactions on Industrial Electronics, vol. 58, pp. 158-172, Jan. 2011.

[8] J. W. Simpson-Porco, F. Dörfler, and F. Bullo, "Voltage Stabilization in Microgrids via Quadratic Droop Control," IEEE Transactions on Automatic Control, vol. 62, pp. 1239-1253, Mar. 2017.

[9] Q. Zhong and G. Weiss, "Synchronverters: Inverters That Mimic Synchronous Generators," IEEE Transactions on Industrial Electronics, vol. 58, pp. 1259-1267, Apr. 2011.

[10] S. D'Arco and J. A. Suul, "Virtual synchronous machines Classification of implementations and analysis of equivalence to droop controllers for microgrids," in Grenoble Conference, IEEE, Jun. 2013.

[11] B. Johnson, M. Rodriguez, M. Sinha, and S. Dhople, "Comparison of virtual oscillator and droop control," in Workshop on Control and Modeling for Power Electronics, 2017.

[12] M. Sinha, F. Dörfler, B. B. Johnson, and S. V. Dhople, "Synchronization of Liénard-type oscillators in heterogenous electrical networks," in Indian Control Conference, pp. 240-245, 2018.

[13] M. Colombino, D. Groß, J.-S. Brouillon, and F. Dörfler, "Global phase and magnitude synchronization of coupled oscillators with application to the control of grid-forming power inverters," arXiv:1710.00694, Oct. 2017.

[14] M. Colombino, D. Gros, and F. Dörfler, "Global phase and voltage synchronization for power inverters: A decentralized consensus-inspired approach," in IEEE Annual Conference on Decision and Control, pp. 5690-5695, Dec. 2017.

[15] M. Li, Y. Gui, J. C. Vasquez, and J. M. Guerrero, "Adaptive synchronization of grid-connected three-phase inverters by using virtual oscillator control," in IEEE Applied Power Electronics Conference and Exposition, pp. 1130-1135, 2018.

[16] H. K. Khalil, Nonlinear systems. Prentice Hall, 2002.

[17] T. Kanamaru, "Van der Pol oscillator," Scholarpedia, vol. 2, no. 1, p. 2202, 2007.

[18] B. B. Johnson, M. Sinha, N. G. Ainsworth, F. Dörfler, and S. V. Dhople, "Synthesizing Virtual Oscillators to Control Islanded Inverters," IEEE Transactions on Power Electronics, vol. 31, pp. 6002-6015, Aug. 2016.

[19] M. Sinha, F. Dörfler, B. B. Johnson, and S. V. Dhople, "Uncovering Droop Control Laws Embedded Within the Nonlinear Dynamics of Van der Pol Oscillators," IEEE Transactions on Control of Network Systems, vol. 4, pp. 347-358, Jun. 2017.

[20] M. Sinha, S. Dhople, B. Johnson, N. Ainsworth, and F. Dörfler, "Nonlinear supersets to droop control," in Workshop on Control and Modeling for Power Electronics, 2015.

[21] J. W. Simpson-Porco, F. Dörfler, and F. Bullo, "Droop-Controlled Inverters are Kuramoto Oscillators*," IFAC Proceedings Volumes, vol. 45, pp. 264-269, Sep. 2012.
[22] S. C. Shabshab and D. F. Opila, "Extending control stability results from voltage-source to current-controlled AC or DC power converters," IFAC-PapersOnLine, vol. 49, pp. 60-65, Jan. 2016.

[23] M. Braun, J. Brombach, C. Hachmann, D. Lafferte, A. Klingmann, W. Heckmann, F. Welck, D. Lohmeier, and H. Becker, "The future of power system restoration: Using distributed energy resources as a force to get back online," IEEE Power and Energy Magazine, vol. 16, no. 6, pp. 30-41, 2018. 